\title{
Germanica
}

$47 \mid 2010$

« Krack ! Tschock ! Pflatsch ! Bummmm ! La BD de langue allemande (à suivre...) »

\section{Splendeurs et misères de quelques homos, hétéros et autres animaux : Comme des lapins de Ralf König ou la comédie de mœurs façon BD}

Glanz und Elend einiger Schwuler, Heteros und anderer Tierarten: Wie die Karnickel von Ralf König oder die comicartige Sittenkomödie.

Splendors and miseries of some homosexuals, heterosexuals and other animals : Like Rabbits by Ralf König as a comic-like comedy of manners.

\section{Hélène Boursicaut}

\section{(2) OpenEdition}

Journals

Édition électronique

URL : http://journals.openedition.org/germanica/1127

DOI : 10.4000/germanica. 1127

ISSN : 2107-0784

Éditeur

Université de Lille

Édition imprimée

Date de publication : 31 décembre 2010

Pagination : 127-144

ISBN : 9782913857261

ISSN : 0984-2632

Référence électronique

Hélène Boursicaut, «Splendeurs et misères de quelques homos, hétéros et autres animaux : Comme des lapins de Ralf König ou la comédie de mœurs façon BD », Germanica [En ligne], 47 | 2010, document 9, mis en ligne le 01 décembre 2012, consulté le 06 octobre 2020. URL : http:// journals.openedition.org/germanica/1127; DOI : https://doi.org/10.4000/germanica.1127

Ce document a été généré automatiquement le 6 octobre 2020.

(c) Tous droits réservés 


\title{
Splendeurs et misères de quelques homos, hétéros et autres animaux : Comme des lapins de Ralf König ou la comédie de mœurs façon BD
}

\author{
Glanz und Elend einiger Schwuler, Heteros und anderer Tierarten: Wie die \\ Karnickel von Ralf König oder die comicartige Sittenkomödie. \\ Splendors and miseries of some homosexuals, heterosexuals and other animals : \\ Like Rabbits by Ralf König as a comic-like comedy of manners.
}

\section{Hélène Boursicaut}

«Goethe war auch ne Sau ${ }^{1}$ »

1 En près de trente ans de carrière, «le (presque) unique bédéraste allemand connu ${ }^{2}$ " aura tracé son chemin pour devenir sinon un vétéran, qualificatif que Ralf König récuse vertement $^{3}$, du moins un incontournable, voire un classique de la bande dessinée allemande, dont on signalera au passage qu'il est traduit dans quinze langues et lu dans dix-sept pays.

Celui qui n'a jamais fait mystère de son homosexualité, sans pour autant se définir comme un militant de la cause gay ${ }^{4}$, a entre-temps élargi son horizon. Se cantonnant à ses débuts dans l'étude du milieu homo de Cologne, dont il se fait le chroniqueur avec les quatre volumes des Gai Comix (Schwulcomics, 1981-86) et surtout la série consacrée au couple Conrad et Paul, s'étendant de 1992 à 2005, Ralf König connaît la consécration avec Les nouveaux mecs (Der bewegte Mann, 1987), ouvrage qui sera adapté pour le cinéma en 1994 par Sönke Wortmann et donnera à la comédie allemande un nouveau souffle. À partir de là, son œuvre se diversifie: parodie du polar noir avec La capote qui tue (Kondom des Grauens, 1987), également porté à l'écran, et Le retour de la capote qui tue (Bis auf die Knochen, 1990), transposition humoristique d'œuvres de la littérature universelle comme Lysistrata (idem, 1987), d'après une comédie d'Aristophane, Iago (Jago, 1998), d'après plusieurs pièces de Shakespeare, voire réécriture fantaisiste de la Bible avec 
Prototype et Archétype (Protyp, Archetyp), deux séries parues entre 2007 et 2009 dans la Frankfurter Allgemeine Zeitung. Déjà lauréat du prix Max-und-Moritz en 1992, Ralf König reçoit en 2006 le prix spécial du jury pour sa prise de position en faveur de la liberté de la presse dans le cadre de la controverse suscitée par les caricatures de Mahomet. De fait, dans les tout derniers albums comme Djinn Djinn (Dschinn Dschinn. Der Zauber des Schabbar, 2005, et Dschinn Dschinn 2. Schleierzwang im Sündenpfühl, 2006), l'auteur poursuit sa satire de l'homophobie, mais en s'en prenant cette fois à la religion et plus spécialement à l'islamisme intégriste.

3 Si nous avons choisi de nous pencher sur Comme des lapins (Wie die Karnickel, 2002), qui a obtenu en 2005 le prix du scénario du Festival International de Bandes Dessinées d'Angoulême, c'est que cet album reflète bien l'évolution de l'œuvre de Ralf König. Ce dernier y dépasse en effet la thématique exclusivement gay pour conter les tribulations amoureuses de l'hétérosexuel Horst Bömmelburg et celles de l'homosexuel Siegfried Purzmann. Ces deux histoires croisées deviennent ainsi le prétexte à explorer avec humour et sans tabou la complexité des relations entre hommes et femmes, mais aussi hétéros et homos. Sur cette étude de mœurs vient en outre se greffer l'observation lucide d'un environnement sociétal qui, aux yeux de l'auteur, fait tout pour formater l'individu.

4 La contribution qui va suivre combinera approche stylistique, étude des personnages et analyse thématique pour tenter de montrer que Ralf König a su renouveler le genre de la comédie de mœurs en la mariant au neuvième art. Une attention particulière sera accordée pour finir à quelques aspects de la traduction française.

\section{Richesse narrative et minimalisme graphique}

Contrairement aux Nouveaux mecs, Comme des lapins est d'abord un film dont Ralf König écrit le scénario en $2002^{5}$ avant de l'adapter sous forme de bande dessinée, laquelle existe elle-même en deux versions, la première parue chez Achterbahn en $2002^{6}$, la seconde l'année suivante chez Rowohlt? ${ }^{7}$.

6 S'éloignant de Cologne où il réside et dont il a fait le décor de la plupart de ses albums, l'auteur situe cette fois l'intrigue, qui se déroule sur quelques mois, à Munich. Lorsque démarre l'histoire, les relations entre Horst, contrebassiste à l'orchestre symphonique de Munich, et Véra, assistante maternelle, ne sont guère au beau fixe. Le jour où sa compagne découvre que Horst regarde en cachette des cassettes pornographiques et décide de le quitter pour retourner chez sa mère, emménage un nouveau voisin de palier, Siegfried dit Sigi, homosexuel libéré, qui vient lui-même de rompre avec son ami Hubertus, dit Hubert. Les deux hommes, qui découvrent qu'ils sont d'anciens camarades d'école, vont rapidement sympathiser, chacun s'initiant progressivement et parfois non sans difficultés au monde de l'autre tout en vivant en parallèle leurs histoires amoureuses.

7 À partir de ce «choc des cultures ", qui constituait déjà le ressort de l'album Les nouveaux mecs, Ralf König propose deux bandes dessinées qui étoffent passablement le scénario cinématographique, comme il le note lui-même dans la postface de la première version : «[...] comme en dessinant ma créativité s'est évidemment une fois de plus emballée, il y a dans l'album des scènes et des personnages qui ne sont pas dans le film ${ }^{8}$.» Et il faut croire que c'est son imagination débordante qui lui a fait reprendre l'histoire, l'album paru chez Rowohlt constituant une version non seulement revue, 
mais aussi élargie du premier jet. Hormis l'ajout de douze pages, les modifications portent essentiellement sur la composition. Dans la première version, des récitatifs structurent le récit et permettent à la voix off de clarifier la situation sous forme de résumés ou d'annonces, voire de révéler et de commenter sur un ton faussement sentencieux les états d'âme et les pensées les plus intimes des deux héros, c'est-à-dire d'orienter et de diriger le lecteur. À une exception près (le récitatif introductif), ceux-ci ont été remplacés dans la seconde version par des dialogues supplémentaires. La continuité linéaire du récit est, ce faisant, abandonnée au profit d'un découpage séquentiel, les scènes se succédant et s'enchainant sans transition ni temps mort, ce qui a pour effet de dynamiser l'intrigue.

8 Autre changement: dépassant l'histoire proprement dite, la première version se projette dans l'«avenir radieux » des deux protagonistes, imaginant pour Horst une autre vie avec une nouvelle compagne sexuellement libérée, ne répugnant comme lui ni à l'adultère ni à l'échangisme, et, en contrepoint, pour Siegfried une relation monogamique avec un militant de Greenpeace au fin fond de la forêt bavaroise, qui plus est avec trois enfants adoptés (sic!), ce qui a pour effet d'inverser complètement le caractère des deux personnages et, partant, la notion de «normalité ». En revanche, la seconde version fait en quelque sorte l'impasse sur le devenir de nos deux héros, les abandonnant chacun à l'émoi d'une nouvelle rencontre, pour sigi celle d'un homosexuel baraqué, velu et tatoué, qu'il s'empresse d'aller retouver au stand de charcuterie, tandis que Horst, au rayon fromages cette fois, fait la connaissance d'une célibataire sexy, qui l'invite à venir prendre le café chez elle et même à regarder un film pornographique en sa compagnie. Tout se passe comme si l'auteur abandonnait désormais ses personnages à leur destin ou, plus exactement, comme s'il abandonnait leur destin à l'imagination du lecteur. A contrario, le passé de Horst et Siegfried, évoqué dans les deux versions par le truchement d'un dialogue des deux personnages sur le thème " du bon vieux temps ", devient, dans la version ultérieure, le point de départ d'un flash-back qui sert en quelque sorte de préface au récit. Cette " pré-histoire », qui plonge le lecteur trente ans plus tôt au sein d'un paisible village bavarois, lui fait faire la connaissance des deux protagonistes enfants qui, sur le chemin de l'école, découvrent leur sexualité. Cette analepse se veut en même temps une clé pour la compréhension des deux personnages dans la mesure où elle met l'accent sur leurs différences : l'un, Horst, bon élève, fasciné par les filles, mais timide et inhibé, l'autre, Sigi, plutôt fainéant, indifférent au beau sexe (mais pas à la taille du pénis de ses camarades ni au charme du professeur de sport!) et déjà déluré, puisque c'est lui qui offre à Horst sa première revue pornographique, dénichée parmi les ordures. La «trouvaille» de Sigi, qui fera office pour Horst de révélation, peut être considérée rétrospectivement comme une prémonition de la future cassette pornographique retrouvée par Véra dans la poubelle au début de l'histoire et à l'origine de la rupture entre les deux partenaires.

9 Comme on le constate, le leitmotiv fait partie intégrante des procédés narratifs de Ralf König. Lorsque s'ouvre le récit, Horst et son collègue musicien et ami Günther sont confortablement installés dans le salon de ce dernier en train de regarder un documentaire sur la vie sexuelle des lapins du désert californien, ce qui donne lieu à une réflexion presque philosophique sur la différence entre l'homme et l'animal et la nécessité, selon Günther, d'un " contrôle social » chez l'être humain : « imagine-nous au niveau des lapins ${ }^{9}$ ", argumente-t-il face à un Horst plutôt partisan d'un retour à une sexualité des plus primitives et qui n'hésite pas du reste à affirmer que même « Goethe 
était un vieux $\operatorname{cochon}^{10}$ ». La formule " comme des lapins ", qui donne aussi son titre au film ${ }^{11}$ et aux deux albums, devient dans l'histoire le mot d'ordre d'une sexualité sans tabou, associée aux yeux de Véra et dans un sens négatif à la pornographie (cf. cdl, p. $\left.41^{12}\right)$. Chez Sigi en revanche, la formule est synonyme de liberté, voire d'hédonisme : Non seulement ce dernier a recours à la comparaison lorsque, se promenant en compagnie de Horst, il lui montre en passant un couple de gays folâtrant dans un buisson - «C'est le printemps! Les hormones explosent! Tout le monde est excité comme des lapins ${ }^{13}$ !! » (Cdl, p. 113) -, mais on peut même voir, dans la scène citée, de vrais lapins en train de batifoler dans les arbres. Qui plus est, on remarque sous le titre des deux versions deux lapins anthropomorphisés représentant l'un Horst avec ses lunettes, le regard triste et le membre pendouillant, l'autre un Sigi hilare au pénis frétillant $^{14}$. Enfin, lorsque Horst, à la fin de l'histoire, fait la rencontre d'une nouvelle compagne au supermarché, vivant cette fois réellement une scène qu'à la suite du documentaire animalier il s'était contenté d'imaginer en s'abandonnant à ses fantasmes, autrement dit en se représentant lui-même en lapin mâle débridé, l'ouvrage, dans la seconde version, se termine justement sur l'image du nouveau couple affublé d'oreilles de lapin.

La richesse narrative de l'album va de pair avec un graphisme épuré, sinon minimaliste. Exception faite de l'illustration et de la quatrième de couverture, l'auteur a opté pour le noir et blanc. De même, il renonce volontiers aux décors. On ne trouvera donc dans les deux versions que trois vues plus ou moins identiques de Munich, faisant penser à une représentation de carte postale, quelques images de l'immeuble où habitent Horst et Sigi, l'aperçu d'un restaurant italien et deux vignettes représentant l'une la maison style chalet tyrolien de la mère de Véra et l'autre la villa où réside la cantatrice bulgare qui s'est entichée de Horst. Le seul espace extérieur ayant véritablement des contours est l'inévitable sex-shop que fréquente Horst et que l'on retrouve presque systématiquement d'un album à l'autre, le pendant féminin en étant le magasin de lingerie où se rend Véra, à la recherche de dessous affriolants pour reconquérir Horst. Pour le reste, les personnages semblent vivre dans des lieux clos, ce qui explique que les vignettes se passent souvent de tout décor ou se contentent d'un mobilier réduit au strict nécessaire (canapé, chaise ou lit), mais favorisant le dialogue. De manière analogue, ce sont le plan moyen, le plan rapproché, le gros plan, voire l'insert qui dominent. Ralf König n'a d'ailleurs jamais fait mystère que ce qui l'intéresse, c'est de «raconter des histoires, sans avoir besoin de beaucoup décrire ${ }^{15}$.» C'est donc la narration qui l'emporte sur la description, celle-ci étant elle-même portée par des personnages hauts en couleur, un sens inné du dialogue et un véritable talent pour le comique de situation.

\section{Des personnages hauts en couleurs}

11 "Quand il parle de ses personnages, König les appelle affectueusement "les nez" ${ }^{16}$.» Hommes ou femmes, tous sont de fait affligés d'un "gros pif », surmonté d'yeux en forme de boule de billard. À partir d'un modèle physique unique, l'appendice nasal contaminant du reste la forme des pieds, eux aussi grands et gros, l'auteur donne forme et vie à toute une constellation de personnages que l'on serait tenté de qualifier de types particularisés dans la mesure où, tout en ayant une valeur d'exemplaires, ceux-ci sont également pourvus d'une personnalité qui leur est propre. Il faut en outre noter 
que l'auteur affectionne les duos, peuplant son univers de couples complémentaires ou antagonistes dont la relation, la plupart du temps, est perturbée par la présence d'un tiers, et qu'il aime, si l'on peut dire, à croiser les « espèces » : être humain ou animal, homme ou femme, hétéro ou homo, on retrouve à tous les niveaux le modèle du doublet. 
Annexe

Wie die Karnickel/Comme des lapins en images ( $\mathcal{O}$ tous droits reservés)

1. Illustration de couverture

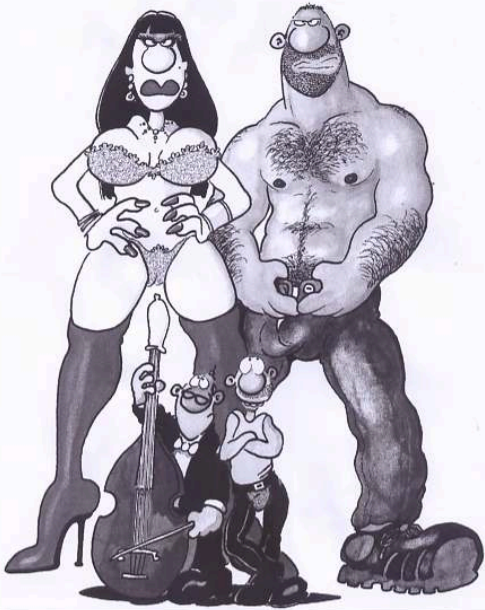

.

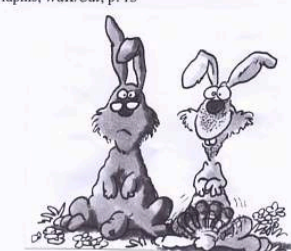

3. Horst et Günther commentant le documentaire sur la vie sexuelle des lapins du déser

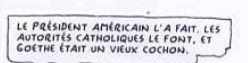

(4).7)

(turii)

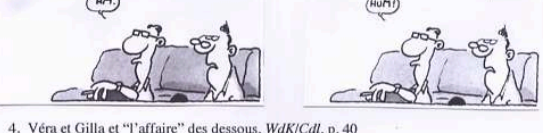

4. Véra et Gilla et "l'affaire" des dessous, WdK/CCl, p. 40

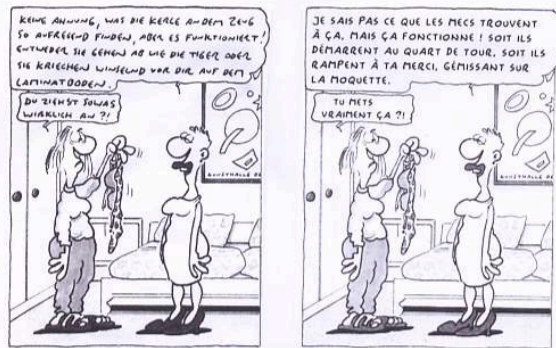




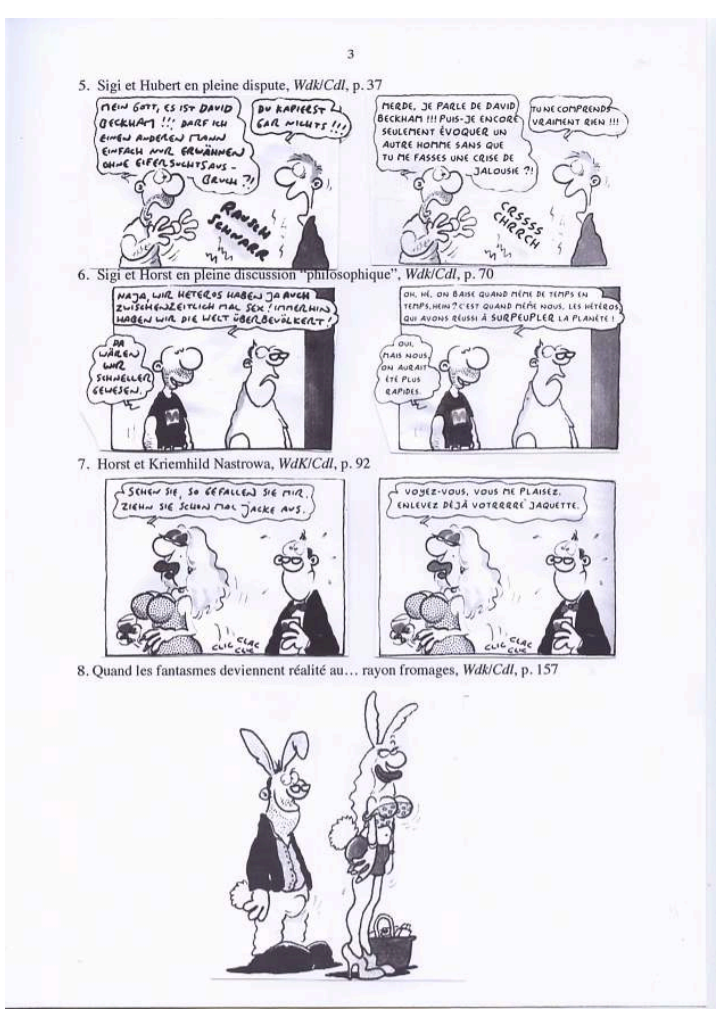

Des femmes de l'histoire, autant dire d'emblée que Ralf König ne les gâte pas, comme à son habitude, ce qui lui a d'ailleurs souvent valu des reproches de misogynie contre lesquels il s'est toujours défendu en arguant d'un important public féminin ${ }^{17}$. La gent féminine se divise grosso modo en deux catégories. Il y a d'abord le type de la grincheuse, incarnée par Véra, la compagne de Horst, remarquablement interprétée dans le film par la comédienne Anna Böttcher. Le genre de la bande dessinée permet à Ralf König de forcer encore le trait : informe, le cheveu pendouillant, sans une once de maquillage, les seins tombants, Véra est le type même de la future épouse ennuyeuse, dénuée du moindre sens de l'humour et sans goût aucun pour les ébats sexuels auxquels aspire son compagnon. Le récitatif introductif souligne ainsi tout ce qui la sépare de Horst : «Véra voudrait se marier. Pas Horst. Véra voudrait des enfants, pas Horst. Véra et Horst sont en période de crise, sexuelle comme en général ${ }^{18}$.» ( $c d l$, p. 16) On ne s'étonnera donc guère que Horst soit attiré par l'exact contraire. À l'autre extrémité de la "planète » des femmes königiennes, on trouve en effet la pin up, à la poitrine généreuse, au maquillage outrancier, adepte autant de hauts talons que de dessous très suggestifs et surtout bête de sexe. Encore faudrait-il préciser que cette version de la femme fatale se décline en de multiples exemplaires, puisqu'elle va de l'actrice de films X siliconée Samantha Whopper ${ }^{19}$ sur laquelle fantasme Horst à l'extravagante cantatrice bulgare Kriemhild Nastrowa, Castafiore königienne à l'accent roucoulant et nymphomane qui croise la route de Horst, en passant par l'amie de Véra, Gilla, qui ne répugne pas à s'encanailler pour plaire à son mari et la jeune femme sexy en diable que Horst rencontre à la fin de l'histoire. Entre la « bobonne gnangnan » et la "bombe sexuelle", les lesbiennes, qui, selon le mot de Sigi, «ne ressemblent pas vraiment à Samantha Whopper, [mais] plutôt à Constantin Wecker avec des seins ${ }^{20}$ " (Cdl, p. 79), peinent à trouver leur place, cantonnées qu'elles sont dans des rôles de déménageuses choc ou de féministes obtuses. 
$13 \mathrm{Au}$ rayon hommes, l'éventail proposé est plus large. Contrairement aux premiers ouvrages, l'album Comme des lapins fait la part belle aux hétéros, incarnés par Horst et son ami Günther, tout en en donnant une vision ironique. Et il est vrai que sous la plume de Ralf König et vu d'un point de vue strictement homosexuel, ces derniers sont présentés comme des êtres passablement inférieurs, ce dont témoigne un échange de répliques entre Horst et Sigi, au demeurant repris pour la quatrième de couverture : "Oh, hé, on baise quand même de temps en temps, hein ? C'est quand même nous, les hétéros, qui avons réussi à surpeupler la planète! - Oui, mais nous on aurait été plus rapides $^{21}$.» (Cdl, p. 70) Curieusement, les hétérosexuels sont les seuls binoclards de l'histoire : tandis que Günther se caractérise par d'épaisses lunettes d'intellectuel qui conviennent bien à son caractère pédant, Horst, quant à lui, porte des lunettes demilune qui lui donnent un air perpétuellement ahuri. Horst le presbyte apparaît de fait comme un être timide, complexé, obsédé par le sexe, mais sans grande expérience réelle. La seule chose qui le sauve, si l'on ose dire, est un membre viril impressionnant qui fait toute l'admiration de Sigi. C'est d'ailleurs grâce à la rencontre avec Sigi, qui prend en main l'éducation sexuelle de Horst, que ce dernier va réussir au fil des pages à s'émanciper.

C'est néanmoins dans la peinture des homosexuels qu'excelle Ralf König. Avec le couple formé par Sigi et son ex-ami Hubert, l'auteur renoue avec la veine de ses débuts, Sigi et Hubert n'étant sur tous les plans rien d'autre qu'un remake du couple Conrad et Paul. Il est du reste à noter que lors de la pendaison de crémaillère organisée par Sigi, Paul, sosie de Sigi, mais en deux fois plus petit, débarque de Cologne pour faire une brève apparition, bel exemple s'il en est d'autoréférentialité. Comme Conrad et Paul, Hubert et Sigi sont aussi différents l'un que l'autre, ne fût-ce que sur le plan physique. Tandis que Hubert, nonobstant le traditionnel gros nez, ressemble au gendre idéal, Sigi cultive le look négligé, crâne rasé, barbe de deux jours, pantalon de cuir et tricot de corps. Alors que Hubert dispense des cours de tantrisme qui, soit dit en passant, tournent vite à la leçon d'éducation sexuelle, Sigi gagne plus ou moins bien sa vie comme garçon de café dans des bars gays. Autant l'un, amateur de musique classique, est raffiné, autant l'autre aime la blague et la fête, autant l'un est un maniaque du rangement, autant l'autre n'aime et ne vit que dans le désordre. Mais surtout, Hubert est monogame et terriblement jaloux, alors que Sigi, extraverti, raffole des revues et des vidéos pornographiques et ne dissimule pas son penchant pour les homos musclés, velus, peu loquaces et bien membrés à défaut d'avoir un cerveau. On l'aura compris, selon un procédé de multiplication et de variation cher à Ralf König, Hubert, bien sous tous rapports, mais profondément ennuyeux, n'est finalement qu'un double de Véra, tandis que Sigi incarne la version homosexuelle de Horst, mais un Horst assumant cette fois pleinement ses fantasmes. Du reste, comme dans le cas du couple Véra/Horst, c'est aussi une tierce personne qui fait voler en éclat le duo Sigi/Hubert, l'actrice de films X Samantha Whopper dont rêve Horst trouvant son équivalent en la personne d'un déménageur, Benno, qui ne dédaigne pas de rendre certains "services » à Sigi : inutile de préciser que ces deux «briseurs de ménage » sont à l'origine de situations aussi embarrassantes qu'hilarantes et souvent proches du vaudeville.

15 Autour des protagonistes gravite toute une série de personnages secondaires qui viennent enrichir le monde en vase clos où évoluent ces derniers et contribuent au comique de situation par leurs interventions souvent décalées. Ainsi en va-t-il de la voisine qui vient sonner chez Horst et Véra parce qu'elle a découvert dans la poubelle 
réservée au papier un sac de plastique contenant la fameuse cassette vidéo, celle-là même qui va déclencher le "drame ", ou de la femme de ménage du sex-shop, épiant Horst enfermé dans une cabine vidéo à travers la cloison. On mentionnera encore le personnage de la vendeuse comme celle du magasin de lingerie qui, face à une Véra cramoisie, n'hésite pas à crier à tue-tête à travers tout le magasin : "Erikaaaa! Les 'dessous coquins' de chez 'style meuf', c'est du synthétique ${ }^{22}$ ?» (cdl, p. 57). La palme du comique revient néanmoins à la scène du déménagement de Sigi, réalisée par l'entreprise « arc-en-ciel » (sic !), spécialisée dans les transports gays et lesbiens : tandis que les deux déménageuses, homasses à souhait, suent sang et eau, l'un des employés, très efféminé lui, se contente de porter une plante d'appartement, ce qui nous vaut un dialogue particulièrement savoureux :

- Dites donc, vous croyez qu'on aura fini à 7 heures? Parce que j'ai un cours de danse et on en est au tcha tcha tcha...

- Si en plus des primevères tu prenais aussi un livre ou un $C D$ sous le bras, on aurait peut-être une chance d'y arriver !!

- Je te l'avais dit dès le départ, on aurait dû engager que des lesbiennes ${ }^{23} !(\mathrm{Cdl}$, p. 54)

Personnages principaux ou secondaires, tous les acteurs ou simples figurants de l'histoire ne se contentent pas d'illustrer bien des travers et ridicules humains dont l'auteur se moque toujours avec tendresse, mais reflètent aussi les rêves et les aspirations qui viennent se heurter à la complexité des rapports humains et à la réalité de l'environnement sociétal.

\section{Une peinture de mœurs drôlatique}

On l'aura compris, le sexe est l'une des préoccupations et des obsessions majeures des héros de Ralf König, et il est omniprésent dans l'album, que ce soit à travers le pastiche des films X, révélant au passage une connaissance parfaite du genre, les scènes de copulation récurrentes, entre Sigi et son ex-ami ou son déménageur, entre Horst et sa cantatrice bulgare, ou de simples gags que l'on retrouve d'un ouvrage à l'autre, en particulier le sexe en érection (celui de Horst entre autres) ou en pleine action.

Il serait néanmoins abusif de réduire Ralf König au statut d'auteur de BD pornographiques, tant l'auteur excelle à allier les fantaisies sexuelles, il est vrai crûment dessinées ou commentées, et l'humour. Ce serait de surcroît faire l'impasse sur le talent d'un observateur hors pair de la nature humaine et de la société qui lui a valu le surnom de «Bretécher gay ». Et il est vrai qu'avec sa consœur dont il ne renie pas le patronage, puisqu'il la cite à deux reprises parmi ses références dans son autobiographie, Et en plus il est gaucher (Und das mit links ${ }^{24}$ ), réalisée, on s'en serait presque douté, sous forme de bande dessinée, il partage non seulement une prédilection pour le graphisme dépouillé et expressif, mais aussi un humour sociologique. Dans l'album Comme des lapins, Ralf König se moque autant des hétérosexuels avec le personnage de Horst, l'obsédé frustré, que des homosexuels à travers le portrait d'un Sigi qui se montre le plus souvent futile et volage avant de faire son mea culpa. Surtout, il persiste à montrer combien les préjugés respectifs entravent la compréhension de l'autre. Ainsi, lorsque Sigi révèle à Horst lors de leur première rencontre qu'il est gay, Horst ne peut s'empêcher de réagir en disant spontanément: «Oh ! Je suis désolé25.» ( $\mathrm{Cdl}$, p. 46) Inversement, Sigi a depuis longtemps des idées bien arrêtées sur la gent hétéro, dans son esprit forcément « coincée » sur le plan sexuel (cf. 
Cdl, p. 71) ou dont il doute même qu'elle puisse exister. Ne va-t-il pas jusqu'à déclarer en plein public en argumentant avec des catégories sans appel :

... primo: les hommes hétéros ne savent pas comment bien baiser. Deuxio: les hommes hétéros qui savent comment bien baiser sont des homosexuels latents. Tertio: les hommes hétéros vraiment beaux sont tous des homosexuels latents, qu'ils sachent comment bien baiser ou pas ${ }^{26}$ ! (Cdl, p. 146)

König ne se contente pas de disséquer les rapports homosexuels/hétérosexuels tout en œuvrant au rapprochement des "espèces ", de manière plus générale il met en scène les problèmes relationnels auxquels se heurtent les uns et les autres. Dans son autobiographie, il fait du reste remarquer que «franchement, les homos ont bien les mêmes problèmes de cœur et de cul que les hétéros ${ }^{27}$ ». Avec humour et lucidité, il analyse les difficultés à concilier les idéaux d'une société axée sur la notion de couple et les envies et pulsions de tout un chacun, qu'il soit homo ou hétéro. L'illustration de couverture à elle seule illustre ainsi le gouffre qui sépare le rêve de la réalité : sont représentés en tout petit les deux héros de l'histoire, Horst en smoking avec ses lunettes et sa contrebasse, et Sigi en tenue décontractée et les bras croisés. Derrière chacun des deux protagonistes, on aperçoit deux personnages qui font trois fois leur taille et semblent les écraser, derrière Horst, l'actrice porno Samantha Whopper qui alimente les fantasmes de ce dernier, et derrière Sigi un grand baraqué pas rasé et au poitrail poilu. Sur cette insatisfaction qui naît de l'impossibilité de donner corps à ses obsessions vient se greffer l'emprise de la routine qui tue l'amour. Outre la frustration sexuelle, c'est le quotidien qui a raison de la relation entre Horst et Véra, comme de celle entre Sigi et de Hubert, ainsi que le remarque le premier : «Emménager à deux, c'était nul comme idée! On s'était tapé du bon temps et de la bonne baise pendant des années. Et soudain des problèmes d'arrivée d'eau de la machine à laver restée ouverte, de balai de chiottes inutilisé28 !» ( $C d l, p .36)$ Voilà qui rappelle fortement les reproches que Véra, au tout début de l'histoire, adresse à Horst, parce que ce dernier a oublié de descendre... la poubelle (cf. $C d l$, p. 24). Dans un autre style, c'est pour satisfaire ses envies de réussite sociale que l'insatiable diva bulgare rompt avec Horst (au grand soulagement du reste de ce dernier!) pour épouser le chef d'orchestre qui lui ouvrira les chemins de la gloire: «Que crrrois-tu? Je ne peux pas sacrrifier ma carrière pour une contrebasse ${ }^{29}$ !» (Cdl, p. 133), lui fait-elle remarquer pour justifier sa rupture.

Ralf König n'a donc de cesse de dénoncer les conventions de tout ordre qui, selon lui, brident l'individu en ruinant toute spontanéité. Si Véra montre tant de répugnance à renouveler ses sous-vêtements en dépit des conseils de Gilla, c'est, dit-elle, parce qu'elle a été conditionnée par ses lectures féministes, en particulier celle d'Alice Schwarzer ${ }^{30}$ (cf. $\mathrm{Cdl}, \mathrm{p}$. 40). Même la mère très abusive de Sigi, tout en ayant parfaitement accepté l'homosexualité de son fils, se met en tête d'organiser le mariage de ce dernier :

Le problème, explique Sigi à son déménageur, censé jouer le rôle de Hubert, l'exami, c'est que depuis que les pédés et les lesbiennes peuvent se marier officiellement, ma mère a trouvé que je devrais le faire aussi ! Ma mère a sa carte des Verts. Elle trouve qu'ils se sont assez cassé le cul pour faire passer cette loi, alors en retour, elle veut que je passe aussi à la mairie! Elle trouve que, particulièrement en Bavière, c'est une obligation pour les homos, rien que pour emmerder la $\mathrm{CSU}^{31}$. (Cdl, p. 98)

Quand un conformisme en chasse un autre...

On s'en rend compte, Comme des lapins oscille constamment entre réjouissante comédie de mœurs et vision sans concession de la société allemande et de son évolution. Sont 
ainsi passées au crible toutes les formes de ghettoisation possibles, tels le communautarisme gay, comme on l'a vu avec l'évocation des déménagements homos, mais aussi le féminisme dont Ralf König stigmatise les excès : dans une scène pleine d'humour, Ralf König met en scène deux féministes extrémistes et lesbiennes qui, non contentes de se complaire dans un jargon identitaire, transforment radicalement les raisons de la rupture entre Horst et Véra en accusant ce dernier de l'avoir menacée, puis frappée et même violée, avant de proposer de publier l'histoire passablement déformée dans une revue bien évidemment féministe (cf. $c d l, p$.114-117), à ceci près que l'auteur donne pour titre à la dite revue le nom de la femme de Socrate, Xantippe, connue pour sa laideur et son caractère de mégère.

De même, l'album ne fait pas l'économie d'une parodie satirique de la télé-réalité. Le joyeux finale, qui, comme au théâtre, réunit tous les protagonistes, prend en effet la forme d'un show télévisé auquel se rendent Sigi et Horst malgré les réticences de ce dernier, Sigi s'étant mis en tête de faire se rencontrer Horst et son actrice de films fétiche, la fameuse Samantha Whopper. Pour Ralf König, c'est l'occasion d'épingler toutes les ficelles de ce "genre» télévisé: titre racoleur de l'émission en anglais ("Catch your dreams »), nom programmatique de l'animateur (Dietmar Schlammberg), sans-gêne de ce dernier qui s'immisce dans la vie de Horst, choisi soi-disant au hasard dans le public, déballage de la vie privée de Horst, c'est-à-dire de son goût pour la pornographie, pseudo-psychanalyse sur un divan, lecture de l'article consacré à Véra, invitées surprise (deux stars du X, les deux féministes et éditrices du journal Xantippe), empoignade générale face au public, confession publique de Horst ( Je suis un homme avec un cœur grand comme ça. Mais avec deux couilles accrochées en dessous. Désolés2 ", Cdl, p. 153) et... publicité qui vient interrompre l'émission.

24 C'est dire si Comme des lapins brosse un portrait décapant de la société allemande des années 2000, s'ingéniant à croquer les tics d'époque.

\section{Quelques remarques sur la traduction française}

On terminera cette étude par quelques mots sur la traduction française de l'album, due à Fabrice Ricker, qui a traduit tous les ouvrages de Ralf König pour le compte de Glénat. On passera sur deux ou trois fâcheuses erreurs grammaticales concernant l'accord du participe avec les verbes pronominaux et de curieuses coquilles, comme la traduction de Dortmund par Düsseldorf (cf. p. 98), qui retrouve du reste son nom quelques pages plus loin (cf. p. 104), celle de «Volleyball» par «hand-ball» (p. 45), celle de "Horrorvideos" qui ne désignent rien d'autre que des films d'horreur, mais deviennent des « vidéos terrifiantes » (p. 34) ou encore celle de « Hollywood-Schaukel » par «balcon» (p.152), alors qu'on attendrait le terme «balancelle». Mais dans l'ensemble, on ne peut que saluer le travail du traducteur, qui a su rendre le niveau de langue en dépit d'une tendance à accentuer la vulgarité des propos tenus, transpose excellemment les nombreuses onomatopées et sait aussi faire preuve d'ingéniosité pour rendre quelques jeux de mots difficiles: c'est ainsi que le nom évocateur du présentateur de télévision, "Dietmar Schlammberg" devient "Dietmar Apurin » (p. 137) et que la blague grivoise racontée par l'un des invités de Sigi, «Jedenfalls stand ich so vor ihm und sagte: 'Hallo Mr Mecklenburg, darf ich Sie ein bisschen vorpommern ? !' » donne : « ... alors je me poste devant lui comme ça et dis, pardon M. Haut-Rhin, et si je vous palatinais un peu?!»(p. 125). 
On ne peut néanmoins que regretter une certaine incohérence dans la transposition des références culturelles, politiques et sociétales entre notes explicatives à la fin du livre, omission ou francisation. Pour ce qui est des notes, certaines apportent au lecteur des renseignements appréciables, par exemple sur la Müllerstraße à laquelle Sigi fait constamment référence et qui, comme le signale le traducteur, est « une fameuse rue pleine de bars homos à Munich» ( $C d l$, note 3, p. 158). On a plus nettement plus de mal avec la note fournie pour le chant tyrolien « Mer san die lustigen Holzacker - puam... holjoholido holjodeldi», qui est en fait transposé par «C'est nous les gars de la Marine... Laladirladada... (ou dans le même genre...)» (note 17, p. 158) : cas flagrant d'omission, puisque aucune explication n'est donnée sur le yodle, voire de francisation abusive. Autre cas d'omission : les « vieux Cds» (p. 35) que Hubert rapporte à Sigi lors de son installation dans son nouveau logement sont dans l'original "alte Cd's von Gitte ». En supprimant le nom de cette chanteuse de variété d'origine danoise, Gitte Heanning, très populaire dans les années 60 , le traducteur fait l'économie d'une note explicative. On rétorquera sans doute que la référence ne nuit pas à la compréhension du dialogue, à cette nuance près qu'elle dissimule toute une partie de la personnalité de Sigi, amateur de culture kitch. Ce qui gêne surtout dans l'album non seulement le lecteur au fait des réalités allemandes, mais le lecteur francophone tout court, c'est la francisation abusive de termes ou de références ancrés dans la réalité quotidienne allemande. Pourquoi traduire «Lohnsteuerjahresausgleich » par " tiers provisionnel » (p. 17) ou "Schrankwand " par " armoire normande» (p. 55) ? Comment comprendre que l'air de la Chauve-souris que sifflote Horst en rentrant d'une nuit torride avec sa diva, "Glücklich ist wer vergisst... la la la la... », devienne brusquement le début du poème de Du Bellay, « heureux qui comme Ulysse... la la la la... » (p. 94), alors que par ailleurs le traducteur a pris soin de laisser en allemand dans le texte tous les airs d'opérette interprétés par la cantatrice et de les traduire en note? De même, si l'on peut concevoir les difficultés de la traduction dans le domaine culinaire et tolérer que soient proposés pour "Tilsiter " et "Tiroler Bergkäse " les termes "chèvre » et " chaource " (p. 19), on accepte nettement moins facilement que le rayon charcuteries, offrant "Krakauer, Putenaufschnitt, Leberwurst », devienne d'un seul coup un rayon fromages, proposant «emmental et gruyère » (p.19). Et que dire du met préféré de Horst, les fameuses « Königsberger Klöpse », qui se transforment comme par miracle en «bœuf bourguignon » (p. 97)! C'est ainsi non seulement faire l'impasse sur tout un pan de la culture germanique, mais aussi gommer toute différence culturelle. Nous terminerons par deux exemples allant dans le même sens, mais qui frisent le grotesque : lorsque Gilla, commentant la réussite de la cantatrice Kriemhild Nastrowa, fait remarquer que celle-ci n'aurait pas fait carrière si elle avait eu le physique de Witta Pohl, comédienne de télévision déjà d'un certain âge, ayant incarné à l'envi le personnage de la mère courage faisant face à l'adversité, le traducteur change carrément de registre en évoquant «la mère Denis » (p. 74), dont il n'est du reste pas sûr qu'elle soit encore connue des jeunes générations. Le dernier exemple montrera qu'une francisation abusive peut même déboucher sur une traduction incompréhensible: Sigi a dans sa panoplie un livre de cuisine d'Alfred Biolek, animateur de télévision connu pour ses émissions culinaires et un coming out tardif, mais spectaculaire. Sous la plume du traducteur, le livre de cuisine est désormais l'œuvre d'un certain... «Delanoë» (p. 71) : il faudra nous expliquer comment l'actuel maire de Paris est devenu le sosie de Jean-Pierre Coffe... 
Sans doute eût-il été préférable de s'en tenir à un répertoire de notes permettant de comprendre les nombreuses références sans ôter au livre sa germanité. Les erreurs relevées, sans rien enlever du reste au mérite du traducteur, prouvent finalement la difficulté qu'il peut y avoir à transposer un ouvrage aussi ancré dans la culture quotidienne allemande.

\section{Conclusion}

Avec ses personnages aux travers aussi ridicules que touchants, son dosage savant de pornographie et d'humour décapant, mais aussi son observation fine des codes, des " tics et des tocs " de la vie sociale et culturelle allemande, Comme des lapins s'inscrit bien dans une œuvre que Ralf König a lui-même qualifiée d'« Astérix pour les adultes ${ }^{33}$ ».

Sans doute faudrait-il ajouter pour conclure que cet ouvrage, comme les autres, véhicule à sa manière une leçon de morale et de tolérance sans pour autant verser dans le moralisme : hymne à toutes les libertés, à commencer par la liberté de ton, Comme des lapins se veut également être un appel à l'acceptation des différences et, plus généralement, des faiblesses du genre humain. On comprend mieux, à la lecture de l'album, la remarque de Ralf König à propos de son lectorat qui, depuis ses débuts, à largement dépassé le public gay : «il se trouve qu'entre-temps, tout le monde achète mes bédés... les homos, les hétéros, les jeunes, les femmes ${ }^{34} \ldots$ »

\section{NOTES}

1. Ralf König: Wie die Karnickel, Reinbek, Rowohlt, 2003, p. 18. Sauf mention contraire, toutes les citations sont empruntées à cette édition (abréviation utilisée par la suite : $W d K$ ).

2. Éric Polet : « Tous BD », in : Libération, 23.06.2007.

3. Cf. «Ich kann diese Schwuchtelei nicht mehr sehen». Interview mit Ralf König, in: Spiegel online, 14.12.2004:

«Spiegel online: Sie veröffentlichen seit 25 Jahren Comics. Fühlen Sie sich manchmal als Veteran? König: Ach, Veteran... Sie erwischen mich gerade in der Midlife-Krise. Sicher sind 25 Jahre "ne lange Zeit und die Inhalte ändern sich auch. Ich habe wenig Lust, in 15 Jahren immer noch Geschichten zu machen über kleine knollennasige Männchen, die ganz viel rum ficken. Das ist ja dann in meinem Leben auch nicht mehr so. Bedauerlicherweise. Scheiße!».

[«Spiegel online: Cela fait 25 ans que vous publiez des bédés. Avez-vous parfois le sentiment d'être un vétéran?

König: Ah, vétéran... Vous me tombez dessus en pleine crise de la quarantaine. Il est sûr que vingt-cinq ans, cela fait un bail et que du coup les contenus eux aussi changent. Je n'ai pas très envie de continuer dans quinze ans à raconter des histoires de petits bonshommes à gros pif qui passent leur temps à baiser à droite et à gauche. Il se trouve qu'en ce qui me concerne, ce ne sera plus le cas non plus. Malheureusement. Merde!»]

4. Cf. Anke Schipp: «Vom Leben gezeichnet», in: Frankfurter Allgemeine Sonntagszeitung, 04.01.2004: «König hat es nie als seine Aufgabe gesehen, Homosexuellen zu mehr 
Selbstbewusstsein zu verhelfen. Er definiert sich als Schwuler, aber er ist kein Aktivist. Eher sieht er sich als kleines Rädchen, das dazu beigetragen hat, das Thema zu enttabuisieren.»

[« König n'a jamais estimé que sa mission était d'aider les homosexuels à s'assumer en tant que tels. Même s'il se définit comme gay, ce n'est pas un militant. Il se considère plutôt comme un petit rouage parmi d'autres ayant contribué à ce que l'homosexualité ne soit plus un sujet tabou. »]

5. Sven Unterwaldt : Wie die Karnickel, Munich, Contantin Film, 2002.

6. Ralf König: Wie die Karnickel, Kiel, Achterbahn, 2002 (abréviation pour toutes les références à cette édition : WdKA).

7. Cf. Note 1.

8. «Und weil beim Zeichnen natürlich nochmal die Kreativität mit mir durchging, gibt es im Comic Szenen und Charaktere, die im Film so nicht zu sehen sind.», WdKA, p. 148.

9. Ralf König: Comme des lapins. Traduction : Fabrice Ricker, Grenoble, Glénat, 2004 (abréviation utilisée par la suite : $C d l)$, p. 19 : «Stell dir vor, wir hätten diese soziale Kontrolle nicht... Stell dir vor, wir wären drauf wie die Karnickel da.», WdK, p. 19.

10. Cf. note 1 .

11. Dans le making of à la fin du DVD, Ralf König souligne que le titre s'est imposé le plus naturellement à lui et qu'il l'a retenu pour son côté évocateur, dispensant de tout commentaire supplémentaire. Notons tout de même que dans le film, le sujet porte sur la vie sexuelle... des babouins!

12. «In diesem Samantha Whopper-Porno. Das war immer das gleiche, wie die Karnickel!», WdK, p. 41.

13. «Es ist Frühling! Die Hormone bumpern! Die Leute sind drauf wie die Karnickel!!», WdK, p. 113.

14. Cf. $W d K$, p. 15.

15. Spiegel online, op. cit., : «Ich mag es einfach, Geschichten zu erzählen, ohne so viel beschreiben zu müssen.»

16. Anke Schipp: «Vom Leben gezeichnet», op. cit. : «Wenn König von seinen Figuren spricht, dann nennt er sie liebevoll 'die Nasen'.»

17. Cf. Christian Göttner : «Komisch und krank, geil und traurig». Interview mit Ralf König, in : Subway. Stadtmagazin Braunschweig, juillet 1999: «Oft kommt jetzt der Vorwurf, meine Comics seien frauenfeindlich, was aber eigentlich nicht sein kann, da sonst nicht so viele Frauen meine Comics lesen würden.»

[ On reproche souvent à mes bédés d'être misogynes, ce qui franchement ne peut pas être le cas, car sinon, je n'aurais pas autant de lectrices. »]

18. «Sie möchte heiraten, Horst nicht. Sie möchte Kinder kriegen, Horst nicht. Horst und Vera sind sexuell und überhaupt in der Krise.», $W d K$, p. 16.

19. Dans le film, c'est Kelly Trump, star allemande du film X des années 90, qui joue son propre rôle et garde son nom.

20. «Die sehen nicht so aus wie Samantha Whopper... eher wie Konstantin Wecker mit Titten!», WdK, p. 79.

21. «Naja, wir Heteros haben ja auch zwischenzeitlich mal Sex! Immerhin haben wir die Welt überbevölkert!

- Da wären wir schneller gewesen.», WdK, p. 70.

22. «Erika, die Reizwäsche von 'Tussistyle', ist das voll Synthetik?!!», WdK, p. 57.

23. «Sagt mal... Meint ihr, wir sind bis 19.00 Uhr fertig? Weil dann hab ich schwulen Tanzkurs und wir sind grad beim Cha-cha-cha...»

«Wenn du zusätzlich zu der Primel da noch'n Buch oder ne CD untern Arm klemmst, klappts vielleicht!!»

«Ich habe gleich gesagt, wir machen lieber 'ne rein lesbische Spedition!», WdK, p. 54. 
24. Cf. Ralf König : Und das mit links, Hambourg, Carlsen, 2005, p. 63 \& 75.

25. «Oh, tut mir leid.», WdK, p. 46.

26. «... Erstens : Heterosexuelle Männer haben von wirklich gutem Sex keine Ahnung. Zweitens : Heterosexuelle Männer, die von gutem Sex Ahnung haben, sind latent homosexuell. Drittens : sehr attraktive heterosexuelle Männer sind sowieso latent homosexuell, ob sie Ahnung von gutem Sex haben oder nicht!», WdK, p. 146.

27. Ralf König: Et en plus il est gaucher. Traduction de Fabrice Ricker, Grenoble, Glénat, 2006, p. 50 : «Ich meine, Schwule haben mit Liebe und Sex den gleichen Stress wie Heteros auch.», Und das mit links, op. cit., p. 62.

28. «Das mit dem Zusammenziehen war'ne Scheiss-Idee! Vorher hatten wir jahrlang Spass und guten Sex, und dann gab es nur Stress weil der Wasserhahn von der Waschmaschine nicht abgedreht war oder ich mal die Klobürste nicht benutzt hab!», WdK, p. 36.

29. «Was denkst du?! Ich kann nicht Kariärä opfern für eine Mann an Kontrabass!!!», WdK, p. 133.

30. «Ja, aber ich habe meine Alice Schwarzer Lektüre hinter mir!», WdK, p. 40.

31. «Die Sache ist nämlich, seit Schwule und Lesben offiziell heiraten dürfen, meint meine Mutter, ich müsse das auch tun! Weil meine Mutter ist bei den Grünen, und sie meint, nun hätten sie sich in der Partei für uns abgestrampelt, nun müsse ich mit meinem Freund auch zum Standesamt! Sie meint, vor allem in Bayern sei das Pflicht, dass Schwule und Lesben heiraten nur um die CSU zu ärgern.», WdK, p. 98.

32. «Ich bin ein Mann! Und ich habe ein grosses Herz. Aber da sind zwei Eier dran. Tut mir leid.», WdK, p. 153.

33. Anke Schipp : «Vom Leben gezeichnet », op. cit. : “ 'Asterix für Erwachsene', sagt er selbst. »

34. Et en plus il est gaucher, op. cit., p. 50 : «aber inzwischen kaufen sie ja alle meine Comics... Schwule, Heteros, Jugendliche, Frauen... », Und das mit links, op. cit., p. 62.

\section{RÉSUMÉS}

Après avoir écrit le scénario du film de Sven Unterwaldt en 2002, Comme des lapins, Ralf König en a tiré un album de BD qui existe lui-même en deux versions. La première a été publiée la même année chez Achterbahn, l'autre a paru en 2003 chez Rowohlt. C'est pour cette seconde version, revue et élargie que König a obtenu le prix du scénario au Festival International de la Bande Dessinée d'Angoulême en 2005. Comme des lapins marque un tournant dans l'évolution de l'auteur qui, jusqu'à présent, mettait l'homosexualité au premier plan. Avec cet album qui conte les tribulations amoureuses de l'hétérosexuel Hans Bömmelburg et celles de l'homosexuel Siegfried Purzmann, Ralf König élargit l'éventail de ses thèmes et propose une vision plus différenciée de la société et des rapports humains, ce qui lui vaut désormais l'adhésion d'un public plus large. Alliant richesse narrative et minimalisme graphique, cette étude de mœurs qui explore avec humour et sans tabou la complexité des relations entre hommes et femmes, mais aussi hétéros et homos, va de pair avec l'observation sans concession d'un environnement sociétal qui, aux yeux de l'auteur, fait tout pour formater l'individu et l'empêcher de s'épanouir. Croisant l'approche stilistique, l'étude des personnages et l'analyse thématique, la contribution, qui prend en compte les deux versions, se propose de montrer l'originalité d'un auteur, souvent qualifié de «Bretécher gay ", qui a su renouveler le genre de la comédie de mœurs en la mariant au neuvième art. On se penchera pour terminer sur quelques aspects problématiques de la traduction française. 
Aus dem Drehbuch, das er zu der Filmkomödie von Sven Unterwaldt Wie die Karnickel 2002 verfasste, entwickelte Ralf König ein Comicbuch, das wiederum in zwei Versionen vorliegt. Die erste Fassung erschien im gleichen Jahr bei Achterbahn, die zweite 2003 beim Rowohlt Verlag. Für diese überarbeitete und erweiterte Fassung erhielt König 2005 auf dem Internationalen Comicfestival von Angoulême den Preis für das beste Szenario. Wie die Karnickel bedeutet einen Wendepunkt in der Entwicklung des Comiczeichners, der in seinem bisherigen Werk die Homosexualität in den Vordergrund stellte. Mit dieser Comicstory, die die Liebesabenteuer des heterosexuellen Horst Bömmelburg und des schwulen Siegfried Purzmann schildert, erweitert Ralf König sein Themenspektrum und bietet ein differenzierteres Bild der Gesellschaft und der zwischenmenschlichen Beziehungen, was ihm nun auch eine breitere Leserschaft beschert. Diese durch erzählerische Kraft und zeichnerischen Minimalismus gekennzeichnete Sittenstudie, die die komplexen Beziehungen zwischen Mann und Frau sowie Homo und Hetero humorvoll und ohne Tabu untersucht, geht mit der unerbittlichen Beobachtung einer gesellschaftlichen Umgebung einher, die in der Vorstellung des Autors alles daran setzt, die freie Entfaltung des Individuums durch das bestehende Normen- und Rollensystem einzuschränken. Im vorliegenden Beitrag, der beide Fassungen berücksichtigt, soll anhand der Analyse von Stil, Figurenkonstellation und Themenkomplexen die Originalität dieses oft als " der schwule Bretécher bezeichneten Autors gezeigt werden, welcher die Sittenkomödie durch die neunte Kunst erneuern konnte. Schließlich sollen einige problematische Aspekte der französischen Übersetzung untersucht werden.

After writing the screenplay for Sven Unterwaldt's comedy Like Rabbits in 2002, Ralf König drew upon the scenario for a comic book, which is available in two versions. The first one was released the same year by Achterbahn and the other one in 2003 by the Rowohlt publishing house. The latter in its revised and expanded version won the 2005 Angoulême International Comics Festival Prize for Scenario. Like Rabbits constitutes a turning point in the development of the author who has put until then homosexuality to the fore. With this graphic novel which relates with humor and without taboo the love troubles of the heterosexual Horst Bömmelburg and of the homosexual Siegfried Purzmann, Ralf König extends the range of his subjects and proposes a more diverse view of society and human relationships, what gives him now a wider audience. By combining narrative richness with graphic minimalism this study of manners that analyzes the complexity of relationships between men and women, heterosexual and homosexual people goes hand in hand with the uncomprimising examination of a societal environment that in the opinion of the author restricts the individual freedom and fulfillment through rules and standards. Based on a study of the style, the characters and the themes, the paper that takes booth versions into account attempts to show the originality of an author who is often described as « the gay Bretécher ». Indeed he managed to renew life to the traditional comedy of manners through the ninth art. Finally, some problematic aspects of the french translation will be considered.

\section{INDEX}

Mots-clés : Comédie de mœurs

\section{AUTEURS}

\section{HÉLÈNE BOURSICAUT}

Université Rennes II 